\title{
Performance of a mobile car platform for mean wind and turbulence measurements
}

\author{
D. Belušíc ${ }^{1}$, D. H Lenschow ${ }^{2}$, and N. J. Tapper ${ }^{3}$ \\ ${ }^{1}$ Monash University, School of Mathematical Sciences, Melbourne, Victoria, Australia \\ ${ }^{2}$ National Center for Atmospheric Research, Boulder, Colorado, USA \\ ${ }^{3}$ Monash University, School of Geography and Environmental Science, \\ and Cooperative Research Centre for Water Sensitive Cities, Melbourne, Victoria, Australia \\ Correspondence to: D. Belušić (danijel.belusic@monash.edu)
}

Received: 6 December 2013 - Published in Atmos. Meas. Tech. Discuss.: 30 January 2014

Revised: 26 April 2014 - Accepted: 20 May 2014 - Published: 25 June 2014

\begin{abstract}
The lack of adequate near-surface observations of the stable atmospheric boundary layer spatial structure motivated the development of an instrumented car for mobile turbulence measurements. The calibration and validation of the car measurements are performed using controlled field experiments and a comparison with an instrumented tower. The corrections required to remove the effects of the car motion are shown to be smaller and simpler than the corrections for research aircraft measurements. A car can therefore satisfactorily measure near-surface turbulence using relatively low-cost equipment. Other natural advantages of a car, such as the ability to drive on any road at any time of day or night and follow the terrain slope, as well as its low cost of operation, make it applicable to observations of a variety of flow regimes that cannot be achieved with the usual platforms, such as research aircraft or networks of flux towers.
\end{abstract}

\section{Introduction}

Horizontal homogeneity is a frequently used assumption in studies of the atmospheric boundary layer (ABL). It is used out of necessity because a majority of the measurements are taken either at single points or along vertical towers. However, this assumption is rarely met in reality (Vickers and Mahrt, 1997; Patton et al., 2005; Martínez et al., 2010; Kang et al., 2012). This is particularly evident in the stable ABL, where motions from various origins are superimposed at a measurement point and result in complex signals that are often associated with turbulence intermittency (e.g.
Belušić and Mahrt, 2012; Mahrt et al., 2013). One alternative is research aircraft observations, which provide ABL spatial structure and can be used to quantify the effects of horizontal heterogeneity (Hutjes et al., 2010; Metzger et al., 2013). Research aircraft have received considerable attention over the last several decades (e.g. Lenschow, 1986; Tjernström and Friehe, 1991; Williams and Marcotte, 2000; Isaac et al., 2004; Martin et al., 2011), with a wide range of aircraft sizes and configurations used for ABL observations. Recently there has been a focus on instrumenting and using light and unmanned aircraft for ABL research (e.g. van den Kroonenberg et al., 2008; Metzger et al., 2011; Mayer et al., 2012; Vellinga et al., 2013). Although aircraft offer considerable improvements in measuring spatial structure of the ABL, they have a number of limitations that prevent their use in certain situations. One critical case is the stable ABL because it occurs at night, can be very shallow, and has strong vertical gradients that require almost perfectly terrainfollowing flight tracks.

Instrumenting a car with turbulence sensors provides a new capability for measuring low-level horizontal ABL structure that complements existing techniques using aircraft in the following ways:

- Aircraft can be vertically displaced by turbulent motions, resulting in uncontrolled altitude variations. Similarly, aircraft have difficulty maintaining constant height above varying terrain. These height variations, together with mean vertical gradients of atmospheric variables, can result in artificial fluctuations in aircraft time series (e.g. Vickers and Mahrt, 1997). The car-mounted 
instruments are always at the same height above the ground and are not influenced by turbulent motions.

- Aircraft experiments have shown that flying as low as possible is preferable for estimating surface fluxes (Mann and Lenschow, 1994; Mahrt, 1998). The instruments can be mounted on a car within a few tenths of a meter of the ground up to about $4 \mathrm{~m}$ above the ground.

- Low-level aircraft measurements are predominantly restricted to daytime, while a car can measure at nighttime as well. This is critical for observations of the stable ABL.

- Small unmanned aircraft, some of which can fly at night, have limited payload compared to a car.

- Aircraft are much more expensive to deploy. This may result in limited airborne time, which necessitates tradeoffs between the desired spatial coverage and repeated flight patterns needed for reducing the random flux error (Mahrt, 1998).

- Aircraft cannot fly below a certain speed, while a car can operate as either a stationary or a mobile platform.

- Car motions are more constrained and smaller than aircraft motions, resulting in less contamination of the measured wind by platform motions.

Using instrumented cars for atmospheric measurements is not new. Straka et al. (1996) report on the mobile mesonet, a mobile system for observing mesoscale weather phenomena. The mobile mesonet consists of instrumented cars that sample standard meteorological variables, including the horizontal wind vector, every $1 \mathrm{~s}$. Mayr et al. (2002) and Smith et al. (2010) used instrumented cars for measuring atmospheric thermodynamic properties in complex terrain, excluding the wind vector. Raab and Mayr (2008) studied the complex flow in the Sierra Nevada mountains using a similar system, additionally equipped with a two-dimensional sonic anemometer. Gordon et al. (2012) seem to have been the first to install three-dimensional sonic anemometers on a road vehicle. They studied the enhancement of turbulence on highways using an instrumented truck. However, a detailed examination of the general applicability of an instrumented car for measuring ABL mean wind and turbulent fluxes has not been undertaken previously. Here we show that an instrumented car can be used for measurements of the horizontal mean and turbulence structure of the ABL.

\section{Instrumented car measurements}

\subsection{Instrumented frame}

The motivation was to develop a simple low-cost instrumented platform that can be easily mounted on a car. A lattice aluminium frame is attached to roof racks of a car, with

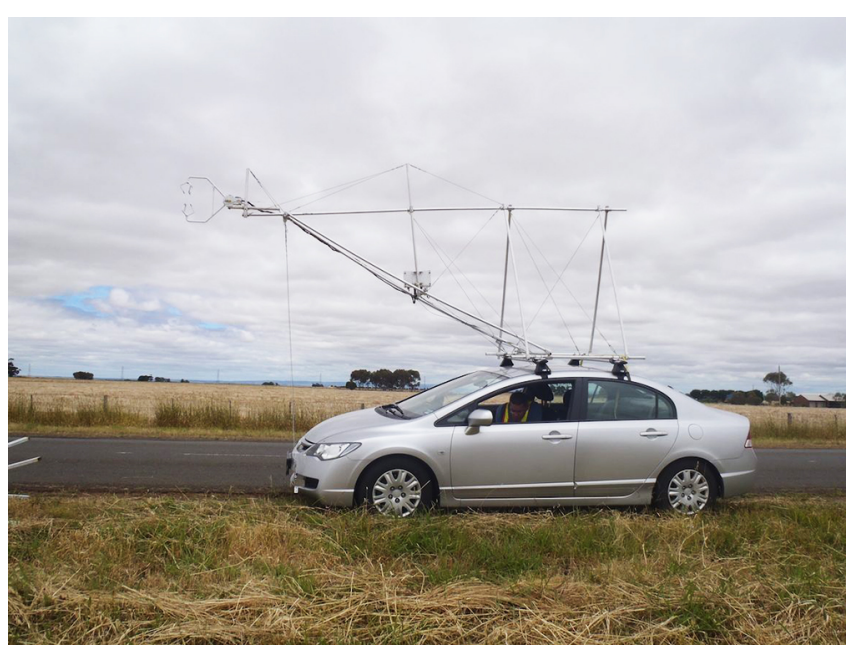

Figure 1. Photo of the instrumented car.

the front of the frame additionally braced to the car bumper with steel guy wires. The latter significantly reduces vibrations of the frame. The frame's attachment point for sensors is located $1.2 \mathrm{~m}$ in front of the car bumper and $3 \mathrm{~m}$ above the ground surface, which is within the legal limits in Victoria, Australia. A CSAT3 sonic anemometer and an unshielded type E fine wire thermocouple (TC) $12.7 \mu \mathrm{m}(0.0005 \mathrm{inch})$ in diameter are used in this system. Figure 1 shows the frame and instrument placement on a car. The sonic and TC data are recorded at $20 \mathrm{~Hz}$. The internal sampling frequency of the sonic is $60 \mathrm{~Hz}$, and this data is internally low-pass filtered and block averaged to $20 \mathrm{~Hz}$ to avoid aliasing. Additional instruments could be installed to measure other variables, such as surface temperature, water vapour, and $\mathrm{CO}_{2}$.

Since the measurement height is $3 \mathrm{~m}$ above the ground and the car typically moves at speeds close to the local speed limits, having the sampling frequency of $20 \mathrm{~Hz}$ raises a possibility of missing the contribution of significant small eddies to the total flux. The latter could also yield increased aliasing effects. Assuming a maximum car speed of $30 \mathrm{~m} \mathrm{~s}^{-1}$, at $20 \mathrm{~Hz}$ this gives a spatial resolution of $\lambda=1.5 \mathrm{~m}$. At the measurement height $z=3 \mathrm{~m}$, we get $z / \lambda=2$. Using surfacelayer scaling (e.g. Lenschow, 1995; Schmitt et al., 1979), we find that more than $95 \%$ of the flux is accounted for at $z / \lambda=2$; e.g. Lenschow, 1995, Fig. 5.4), which means that the current sampling frequency is adequate for the car flux measurements in the daytime. Later we show that the spectra show no signs of aliasing.

A low-cost miniature GPS-aided inertial navigation system (GPS-INS; IG-500N, SBG Systems, France) is attached to the sonic anemometer to provide the position, speed and orientation of the sonic. The GPS-INS data are used to remove the car motions from the sonic measurements, and to rotate the wind vector measured in the car coordinate system to the meteorological coordinate system (defined in Sect. 2.2). The development of small low-cost GPS-aided 
INS has made this type of inexpensive system feasible for turbulence measurements. The somewhat lower accuracy of such systems compared to more expensive airborne INS systems is partially offset by the smaller amplitude of car motions compared to aircraft motions. The GPS-INS data are recorded at $20 \mathrm{~Hz}$. The internal sampling frequency of the accelerometers and gyroscopes is $10 \mathrm{kHz}$, which is downsampled through a series of internal filters to $100 \mathrm{~Hz}$, and then merged with the GPS signal sampled at $4 \mathrm{~Hz}$ through the on-board extended Kalman filter. Since the final output is based on integration of accelerometer and gyroscope data, small initial errors can grow rapidly without external corrections. The GPS is used to provide such long-term stability to the final results. The GPS is also used to provide the heading.

The frame can be attached to a wide variety of cars; a dedicated car is not necessary. In order to simplify the mounting of the frame on a car, the frame is permanently attached to a set of roof racks. It takes two people to carry the frame, and less than $30 \mathrm{~min}$ to attach the roof racks to a car and the guy wires to the bumper. Some instruments are installed after the frame is attached to a car, so it takes about $1 \mathrm{~h}$ to set up the entire system and start the measurements.

\subsection{Determining the wind vector}

The current GPS-INS is factory configured such that it outputs velocity in its own coordinate system, unlike the typical INS systems that output velocity in the geographic coordinate system, with the $z$ axis aligned with the local Earth gravity. The GPS-INS is mounted such that its coordinate system is aligned with the sonic's coordinate system, which are then aligned with the car coordinate system. This means that the wind vector in the car coordinate system is a simple vector difference between the sonic and GPS-INS velocities:

$\boldsymbol{V}_{\text {car }}=\boldsymbol{V}_{\text {sonic }}-\boldsymbol{V}_{\text {INS }}$,

where $\boldsymbol{V}_{\text {car }}$ is the wind vector in the car coordinate system, $\boldsymbol{V}_{\text {INS }}$ is the GPS-INS motion vector, and $\boldsymbol{V}_{\text {sonic }}$ is the the car-relative flow vector measured by the sonic anemometer.

The typical procedure for obtaining the final wind vector from the current system is the following. We assume here that the departures of the car coordinate system from the local terrain coordinate system are negligible. Then the wind vector in the coordinate system defined by the local terrain, $\boldsymbol{V}_{\text {ter }}$, is obtained by rotating $\boldsymbol{V}_{\text {car }}$ horizontally using the heading information:

$\boldsymbol{V}_{\text {ter }}=\mathbf{M}_{\mathrm{tc}} \boldsymbol{V}_{\mathrm{car}}$,

where

$\mathbf{M}_{\mathrm{tc}}=\left(\begin{array}{cc}\cos \Psi & -\sin \Psi \\ \sin \Psi & \cos \Psi\end{array}\right)$

is the two-dimensional rotation matrix, and $\Psi$ is the heading. The final wind vector in the local meteorological coordinate
Table 1. Accuracy of the GPS-INS and sonic, given by the manufacturers, and the derived accuracy of the final wind. $u$ and $v$ for the final wind are evaluated using $u_{\text {car }}=v_{\text {car }}=5 \mathrm{~m} \mathrm{~s}^{-1}$ (see Eq. 3).

\begin{tabular}{llll}
\hline Parameter & GPS-INS & Sonic $\left(@ 20 \mathrm{~m} \mathrm{~s}^{-1}\right)$ & Wind \\
\hline Pitch, roll & $0.8^{\circ}$ & - & - \\
Heading $(\Psi)$ & $0.5^{\circ}$ & - & - \\
$u, v$ & $0.1 \mathrm{~m} \mathrm{~s}^{-1}$ & $0.5 \mathrm{~m} \mathrm{~s}^{-1}$ & $0.6 \mathrm{~m} \mathrm{~s}^{-1}$ \\
$w$ & $0.1 \mathrm{~m} \mathrm{~s}^{-1}$ & $0.4 \mathrm{~m} \mathrm{~s}^{-1}$ & $0.5 \mathrm{~m} \mathrm{~s}^{-1}$ \\
Position & $2.5 \mathrm{~m}$ & - & - \\
\hline
\end{tabular}

system $\boldsymbol{V}$ is obtained from

$\boldsymbol{V}_{\text {ter }}=\left(u_{\text {ter }}, v_{\text {ter }}, w_{\text {ter }}\right)=($ northward, eastward, downward $)$

by a simple permutation of components:

$\boldsymbol{V}=(u, v, w)=($ eastward, northward, upward $)$

$$
=\left(v_{\text {ter }}, u_{\text {ter }},-w_{\text {ter }}\right) \text {. }
$$

Note that eastward, northward, and upward or downward are relative to the local terrain, so that the local vertical is determined by the slope of the terrain rather than by gravity. In this way, the wind vector is given in the coordinate system that is approximately aligned with local flow streamlines, which for stationary sonic anemometers is normally achieved by using tilt correction algorithms (e.g. Wilczak et al., 2001). The alignment with the local road slope is not desirable for small-scale road irregularities that are not followed by the airflow. This could be addressed by applying pitch and roll corrections only to the high-wavenumber part of the spectra, but the results from the field tests discussed below show that these corrections are usually very small. This natural alignment with the local terrain slope is an advantage of the instrumented car compared to research aircraft. Aircraft observations have to be three-dimensionally rotated into the global meteorological coordinate system, with the vertical determined by gravity (e.g. Lenschow, 1986; van den Kroonenberg et al., 2008). While this three-dimensional transformation can be used for the car as well, we perform it only in specific cases, such as in the validation test where the car is driven over speed bumps.

Using the two-dimensional rotation simplifies the determination of the error propagation. The accuracy of the final wind vector is obtained using Eqs. (1) and (2) from the nominal accuracies of the GPS-INS and sonic, which are provided by the manufacturer (Table 1 ), in the following way:

$$
\begin{aligned}
\Delta \boldsymbol{V}^{\mathrm{h}} & =\Delta \boldsymbol{V}_{\text {sonic }}^{\mathrm{h}}+\Delta \boldsymbol{V}_{\mathrm{INS}}^{\mathrm{h}}+\boldsymbol{V}_{\mathrm{car}}^{\mathrm{h}} \Delta \Psi, \\
\Delta w & =\Delta w_{\text {sonic }}+\Delta w_{\mathrm{INS}},
\end{aligned}
$$

where $\Delta$ denotes the error and superscript h the horizontal wind vector.

The systematic error due to potential misalignment between the sonic and GPS-INS can be evaluated by assuming the misalignment angles in the vertical and horizontal 
Table 2. Basic characteristics of the 20 car tracks. $\left|V_{\mathrm{INS}}^{\mathrm{h}}\right|$ is the mean horizontal car speed (see Eq. 1).

\begin{tabular}{lll}
\hline Tracks & $\begin{array}{l}\left|\boldsymbol{V}_{\mathrm{INS}}^{\mathrm{h}}\right| \\
\left(\mathrm{m} \mathrm{s}^{-1}\right)\end{array}$ & $\begin{array}{l}\text { Length of } \\
\text { tracks }(\mathrm{m})\end{array}$ \\
\hline 1 and 2 & 13.2 & 780 \\
3 to 6 & 15.9 & 900 \\
7 to 14 & 21.2 & 900 \\
15 to 20 & 26.8 & 900 \\
\hline
\end{tabular}

planes of $1^{\circ}$. This value is larger than the actual angles because the misalignment is minimized by attaching the GPSINS directly to the head of the sonic. The resulting error in transverse components is less than $0.35 \mathrm{~m} \mathrm{~s}^{-1}$, but this error is mostly removed by the correction procedures described in Sect. 4.1.

\section{Field tests}

Two field tests were performed to detect and correct various sources of errors, such as the flow distortion induced by the car and frame, and to validate the corrected final wind vector. The tests were designed to mimic the typical research aircraft manoeuvres for in-flight calibration (Lenschow, 1986). The range of those manoeuvres that could be performed by the car is limited because many of them depend on the complicated aircraft responses to changing pitch, lift or speed, none of which occur for the car. An additional limitation occurs for the calibrations that require flying in the calm atmosphere above the ABL, which is obviously unachievable for the car. At the same time, these limitations are compensated for by the reduced amplitude and greater simplicity of car motions compared to the aircraft, so the two tests performed here provide enough data for calibrating the car observations.

\subsection{Test 1: repeated passes and tower comparison}

The first test was conducted in a rural relatively flat-terrain area near Shelford, Victoria, Australia on 6 December 2012, from 12:23 to 13:03 LST. The winds were about $1.5 \mathrm{~m} \mathrm{~s}^{-1}$ at $3 \mathrm{~m}$ above the ground, the surface heat flux was positive, and the sky was covered with scattered stratocumulus clouds. The instrumented car passed a tower while driving back and forth along a relatively flat and homogeneous road (see Fig. 2). There were 20 tracks, and the car speeds ranged from 13 to $27 \mathrm{~m} \mathrm{~s}^{-1}$ (see Table 2). The maximum driving time for each track was $1 \mathrm{~min}$. The length of the first two tracks reached only $780 \mathrm{~m}$ because of the lower car speed. The lengths of all other tracks will be limited to $900 \mathrm{~m}$ in further analyses, except for the spectral calculations. The length of $900 \mathrm{~m}$ is chosen because there the road is relatively flat and homogeneous.

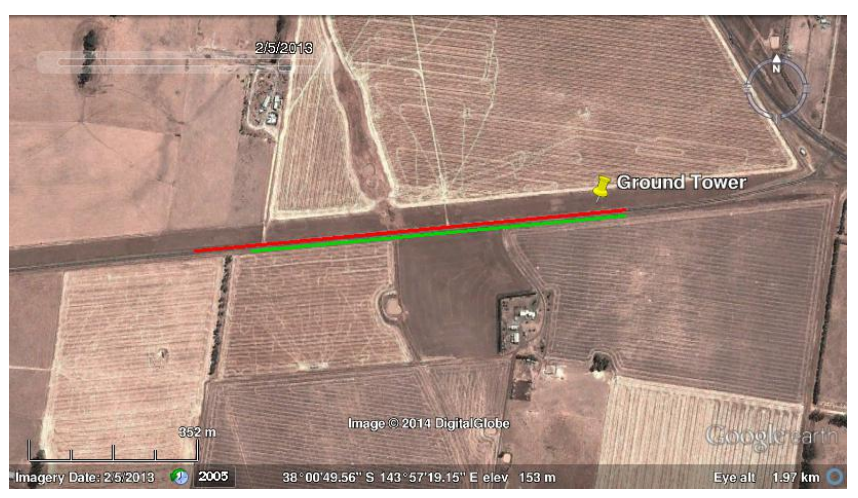

Figure 2. Google Earth image showing the location of the $780 \mathrm{~m}$ (green line) and $900 \mathrm{~m}$ (red line) car tracks, and the instrumented tower.

The tower was equipped with the same instruments as the car (a CSAT3 sonic and a type E fine wire TC with a $12.7 \mu \mathrm{m}$ diameter) at the same height ( $3 \mathrm{~m}$ above the ground) and was located $25 \mathrm{~m}$ from the road near one end of the tracks (Fig. 2).

\subsection{Test 2: speed bumps and small circles}

The second test was conducted on a windy day in Melbourne, Victoria, Australia on 16 August 2013, from 10:30 to 11:30 LST. The wind at $3 \mathrm{~m}$ was around $4 \mathrm{~m} \mathrm{~s}^{-1}$ with strong turbulence and positive surface heat flux. The instrumented car was driven back and forth along a street with several speed bumps. The street is located in a suburban area with a homogeneous grass-covered park upwind. Eight tracks with the total of 27 speed-bump crossings were made, driving at a constant car speed of about $12 \mathrm{~m} \mathrm{~s}^{-1}$.

Another manoeuvre was performed during this test, where the car was driven at a constant speed of about $8 \mathrm{~m} \mathrm{~s}^{-1}$ in small circles, with the radius of $20 \mathrm{~m}$ on the top of a parking garage. The usual corrections failed to remove the car-motion effects from the final wind vector, but were successful when the horizontal car speed was taken from an independent GPS located in the car, rather than from the miniature GPS-INS. A comparison between the two instruments showed that the miniature GPS-INS underestimated the horizontal car speed by about $2 \mathrm{~m} \mathrm{~s}^{-1}$ during this manoeuvre. This could be due to a phase shift between the internal INS and GPS resulting from a time lag in the GPS response, which points to the possible limitation that the current system may not be accurate during sharp turns. As a result, the data for all significant turns will be excluded from the analyses.

\section{Correction and validation of car measurements}

\subsection{Corrections}

The car, the frame and the sensors induce flow distortion. Since the car speeds were about 10 times higher than the true 

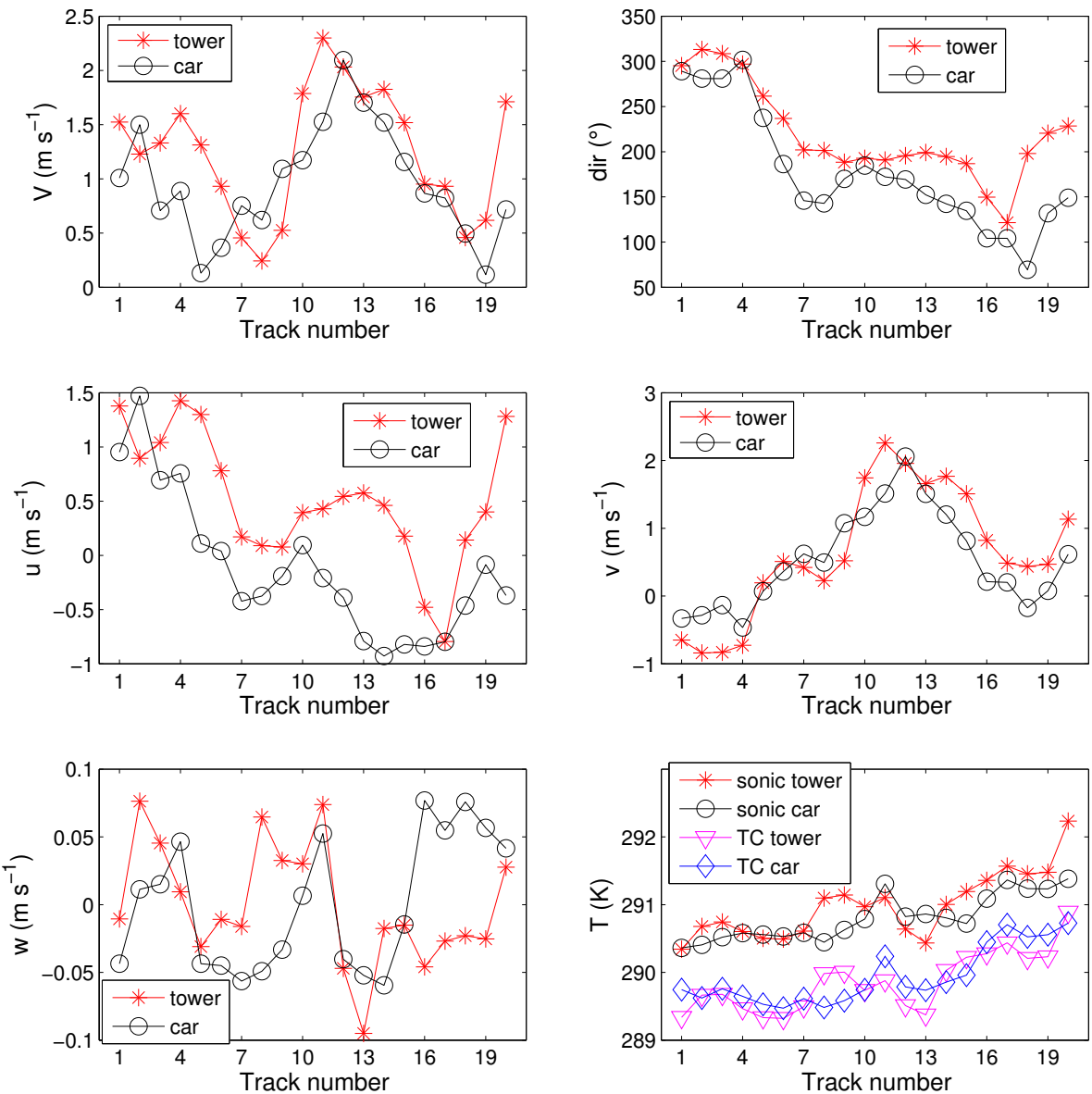

Figure 3. Car vs. tower for mean variables: vector-averaged wind speed $(V)$, wind direction (dir), eastward $(u)$, northward $(v)$ and vertical $(w)$ wind components, and temperature $(T)$.

wind speed, the flow distortion can significantly modify the total measured wind vector and is likely to cause the only significant error in the magnitude of the measured longitudinal wind component. Two corrections were performed in order to reduce the effects of the flow distortion or misalignment of sensors:

- The data show that the vertical wind speed has a linear dependence on the car-relative wind speed (the mean angle of attack is $1.3^{\circ}$ for both tests). This linear dependence is removed by the coordinate rotation of $-1.3^{\circ}$ so that $\bar{w}$ becomes zero for all speeds.

- The horizontal wind speed is corrected by assuming the stationarity of the true wind velocity for any two consecutive tracks driven at same car speeds in opposite directions. If so, the average of the longitudinal car-relative flow speed in the car coordinate system $\left(u_{\text {sonic }}\right)$ for the two tracks should be equal to the car speed. The measured data from the first test shows that the differences between $u_{\text {sonic }}$ averaged over two consecutive tracks and the car speed are a function of the car speed, so they are forced to zero. The same average of the lateral component $v_{\text {sonic }}$ is zero in the data, so a correction is not required. The method applied here is standard in calibrating aircraft measurements. The caveat here is that these manoeuvres are usually performed in the steady atmosphere above the ABL to reduce the effects of nonstationarity and horizontal heterogeneity. These effects may be more important for the car system because of the proximity of the surface. For example, during the $40 \mathrm{~min}$ of the first test, the wind speed oscillated between about $0 \mathrm{~m} \mathrm{~s}^{-1}$ and $2.5 \mathrm{~m} \mathrm{~s}^{-1}$, and the wind direction varied between northwesterly and southeasterly. Based on the tower data, some of the larger wind vector changes occurred between two consecutive tracks, which affected the final correction of the longitudinal component by an unknown amount.

These corrections are applied to all wind data measured by the instrumented car. 


\subsection{Comparison of averages and fluxes with the tower}

Averaging intervals for the car data are taken to be equal to the track lengths, which means that there is a smaller number of data points (i.e. shorter time interval) for tracks with higher car speeds. The reasons for this are the approximate homogeneity of the tracks over the $900 \mathrm{~m}$, and the same spatial scales for calculating turbulent fluxes. The latter is important because there is a significant contribution to the fluxes from the energy-containing region at larger scales. Using the equal track lengths means that the same spatial scales are used for comparison between different tracks and the tower. Changing the track lengths for different speeds (e.g. to force equal time intervals for all tracks) would result in different sizes of large eddies retained in flux calculations, which is undesirable.

For each track, the tower data are averaged over $5 \mathrm{~min}$ for the comparison of mean variables and over $10 \mathrm{~min}$ for the fluxes. The $10 \mathrm{~min}$ averaging for the fluxes is determined by requiring that the same spatial scales, or eddy sizes, are present for both the car and tower fluxes. Since the largest scales in the car measurements are $900 \mathrm{~m}$, and the mean wind speed is about $1.5 \mathrm{~m} \mathrm{~s}^{-1}$ (see Fig. 3), the Taylor hypothesis gives the corresponding timescales of $10 \mathrm{~min}$. The $5 \mathrm{~min}$ averaging for mean variables is chosen instead of $10 \mathrm{~min}$ because the interval of a single car track is 1 min, which means that the car will have driven about six or seven tracks during a single tower average of $10 \mathrm{~min}$. This number decreases to about three tracks when $5 \mathrm{~min}$ averaging is used. Reducing the tower averaging even further would result in insufficient sampling and the data would not be representative of the mean flow. Likewise, consecutive $10 \mathrm{~min}$ tower fluxes have a considerable overlap, yielding smoother flux estimates between different tracks compared to the car. These caveats should be considered when comparing the car measurements with the tower.

Figure 3 shows the comparison between the car and tower mean wind and temperature data for each track. The corresponding scatter plots and statistical measures of agreement (bias and normalized RMSE) are shown in the Supplement. The agreement is satisfactory for all variables, implying that the car can be used for measuring mean horizontal structure of the ABL. The $u$ component seems systematically underestimated by the car, which may be a reflection of the limitations in using a fixed-point wind measurement to compare with the car measurement along a horizontal traverse. The differences are likely due to nonstationarity and horizontal heterogeneity in the wind field. The lack of correlation between $w$ components is expected because of the relatively small magnitude and integral scale of $w$ compared to $u$ and $v$. The sonic anemometer measures the sonic virtual temperature (Kaimal and Gaynor, 1991), which explains the difference from the mean TC temperature in Fig. 3.
Figure 4 shows the comparison between all components of the car and tower fluxes and variances. The corresponding scatter plots, and bias and normalized RMSE, are shown in the Supplement. All three speed variances generally agree well, except for certain tracks (tracks 14 and 15 for $\overline{u^{\prime 2}}$, and tracks 18 and 19 for $\overline{v^{\prime 2}}$ ) that will be discussed below. The car somewhat underestimates the temperature variance for both the sonic and TC, implying that the difference is not caused by instrument errors, but probably by nonstationarities in tower measurements. The car and tower heat fluxes generally follow each other over the range of values, which vary from 0.05 to $0.35 \mathrm{~K} \mathrm{~m} \mathrm{~s}^{-1}$, and the level of agreement for individual tracks depends on the tower averaging interval as shown below. According to expectations (e.g. Schotanus et al., 1983; Foken et al., 2012), the car heat fluxes calculated from the sonic temperature (i.e. buoyancy fluxes) are larger than from the TC temperature (i.e. sensible heat fluxes), but the correlation is good. However, this difference increases with the car-relative flow speed. This is consistent with the overestimation of heat flux by the CSAT3 anemometer in strong winds reported by Burns et al. (2012). They found the source of the error in the underestimation of the sonic temperature by CSAT3 running version 4 of the firmware. We use the same CSAT3 firmware version, and our results are consistent with this documented systematic underestimation of the sonic temperature.

The car momentum fluxes generally are the right order of magnitude, but the agreement on a point-to-point basis is questionable. The car $\overline{v^{\prime} w^{\prime}}$ flux appears as if it has the opposite sign from the tower. The $\overline{u^{\prime} v^{\prime}}$ flux seems to agree the best in that there are no large or systematic discrepancies. However, the car $\overline{u^{\prime} w^{\prime}}$ flux does not look realistic, because the values oscillate around zero for tracks in different directions. Such behaviour could result from two mechanisms: flow distortion that has not been completely corrected, or the random error resulting from insufficient sample size. Tests with several different approaches to correcting the car flow distortion have not changed this behaviour. Li et al. (2013) showed that CSAT3 is prone to erroneous positive momentum flux even with carefully performed sector-specific tilt correction, which could partially explain the unrealistic fluxes obtained here. However, when the averaging interval for the tower fluxes is decreased to $2 \mathrm{~min}$, the tower $\overline{u^{\prime} w^{\prime}}$ fluxes start to exhibit a very similar behaviour to the car (Fig. 5). This is a result of the small sample size for the tower fluxes and the related loss of overlap between subsequent flux estimates. Therefore, a provisional conclusion is that the possible problem with the car $\overline{u^{\prime} w^{\prime}}$ fluxes is predominantly related to the small sample size, rather than to uncorrected flow distortion.

This conclusion is further supported by the analysis of Lenschow and Stankov (1986) and Lenschow et al. (1994), who find that random sampling errors are smaller for scalar fluxes than for momentum fluxes. This is a consequence of the dependence of the random error of fluxes on the 

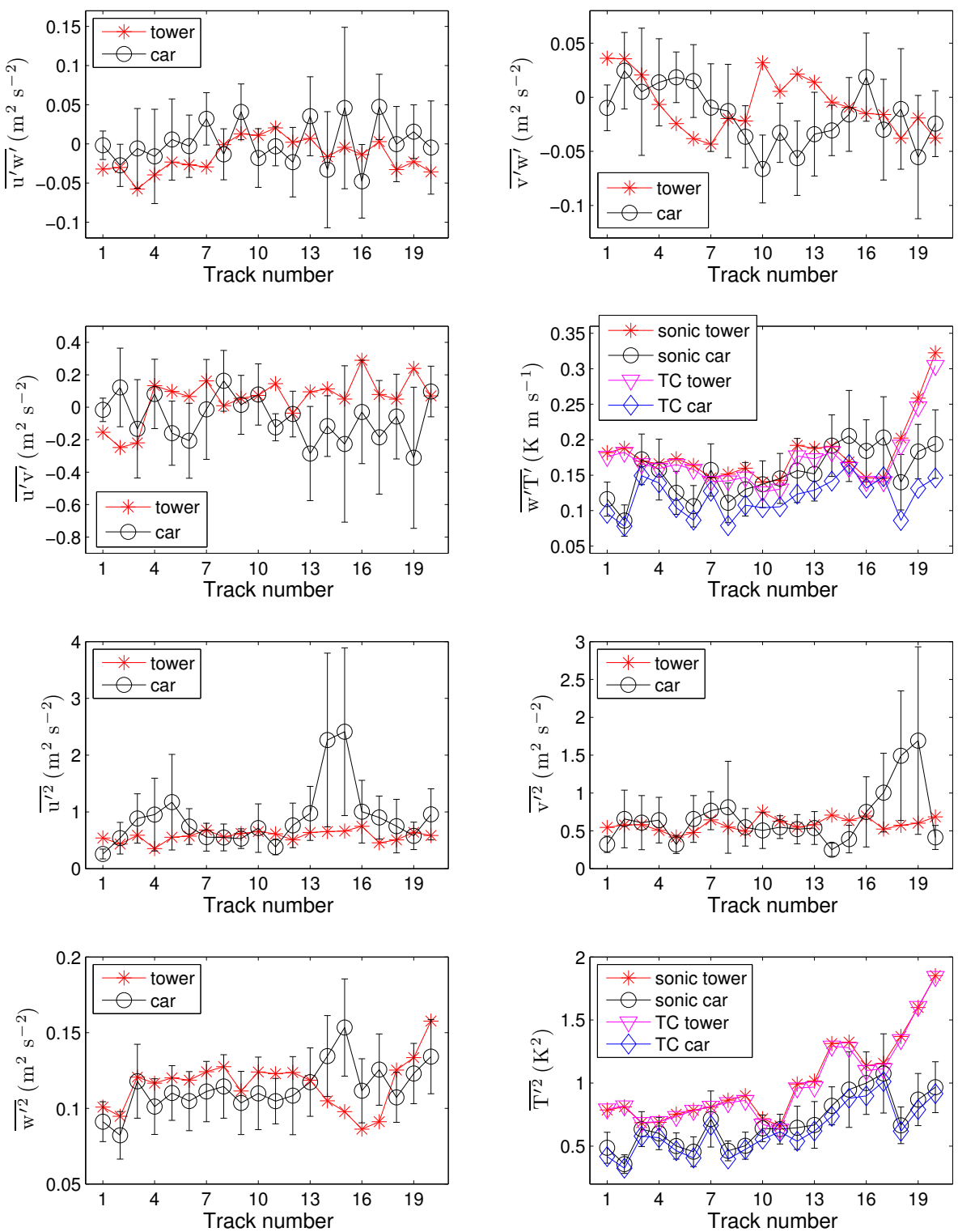

Figure 4. Car vs. tower for fluxes and variances. Averaging interval for the tower data is $10 \mathrm{~min}$. The error bars represent the $95 \%$ confidence intervals $( \pm 1.96 \sigma$, where $\sigma$ is obtained from Eq. (5) for both fluxes and variances).

correlation coefficient (Lenschow et al., 1994):

$$
\left(\frac{L}{2 \mathcal{L}_{\mathrm{f}}}\right)^{1 / 2} \frac{\sigma_{F}(L)}{|F|}=\left(\frac{1+r_{\mathrm{ws}}^{2}}{r_{\mathrm{ws}}^{2}}\right)^{1 / 2},
$$

where $F$ is the flux, $\sigma_{F}$ the random flux error, $\mathcal{L}_{\mathrm{f}}$ the flux integral length scale, $L$ the track length, and $r_{\mathrm{ws}}$ the correlation coefficient between the vertical wind speed $w$ and a variable $s$, where $s$ is either the temperature $T$ or a component of the horizontal wind speed, $u$ or $v$. In this study, $\overline{\left|r_{w T}\right|}=0.55$, $\overline{\left|r_{u w}\right|}=0.07$, and $\overline{\left|r_{v w}\right|}=0.10$, where the overbar denotes the average over all tracks. The term on the right hand side of Eq. (5) is thus 5 to 7 times larger for momentum than for heat flux, while the integral scales are comparable. The integral scale is calculated by integrating the autocorrelation function to the first zero crossing (Lenschow and Stankov, 1986). The magnitudes of random error are given by the error bars in Figs. 4 and 5. The random error for variances is obtained in the same way as for the fluxes, but for the variances, the correlation coefficient is 1 .

Furthermore, the agreement of the other two momentum fluxes is improved when 2 min tower averaging is used, particularly for $\overline{v^{\prime} w^{\prime}}$. The effect of reduced tower averaging on $\overline{w^{\prime} T^{\prime}}$ and $\overline{T^{\prime 2}}$ is also significant, but the differences between tower and car measurements decrease only for some tracks. There is an increase of the tower $\overline{v^{\prime 2}}$ for track 18 that resembles the car $\overline{v^{\prime 2}}$, and it appears only for the 2 min tower 

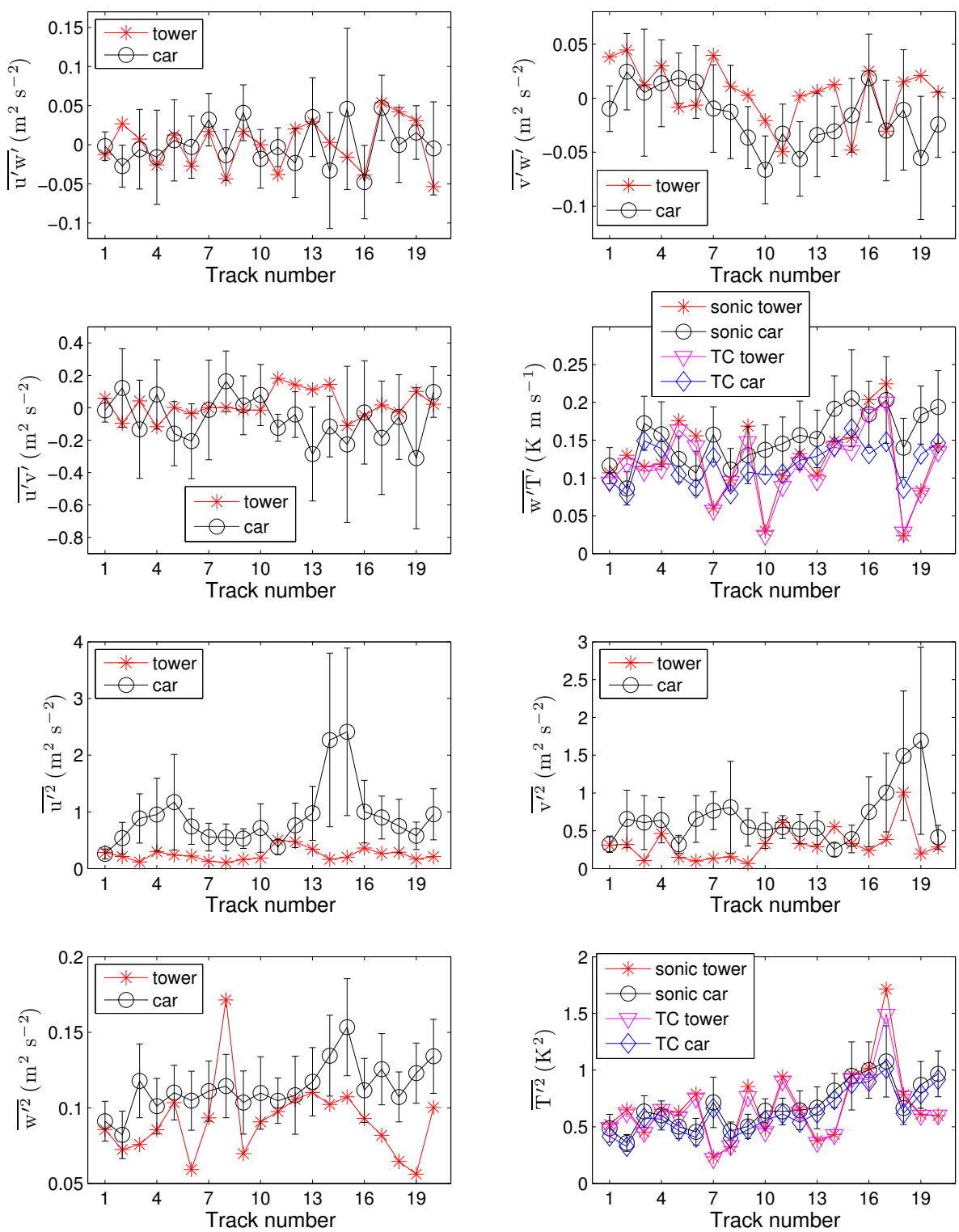

Figure 5. As in Fig. 4, except that the averaging interval for the tower data is $2 \mathrm{~min}$.

averaging. This indicates that the apparently overestimated car variances for some tracks could result from short-lived strong mesoscale events whose magnitudes are reduced by larger averaging intervals for the tower fluxes. Inspection of the raw data for tracks 14 and 15 , where the car considerably overestimates $\overline{u^{\prime 2}}$, points to a similar conclusion that the anomalous variances are due to propagating mesoscale structures that are not adequately sampled by either the tower or the car.

Another cause of differences could be the different footprint for the tower and car. We have calculated the footprint for each car track using the technique described by Hsieh et al. (2000), and the distance of the footprint peak from the car sensors ranges from 2 to $8 \mathrm{~m}$ (Fig. S5 in the
Supplement). The road can therefore exert significant influence on car fluxes in these weak-wind conditions, while the tower is too far away from the road in this case to experience these effects. However, the largest differences between the car and tower are not associated with a noticeable change in the footprint or wind direction, meaning that other mechanisms (discussed above) are most likely responsible for the larger differences.

\subsection{Comparison of spectra with the tower}

The spectra are calculated in the wavenumber domain for the comparison between the car and the tower. The input time series are transformed from the time to space domain using the car-relative flow speed for the car spectra, and the 


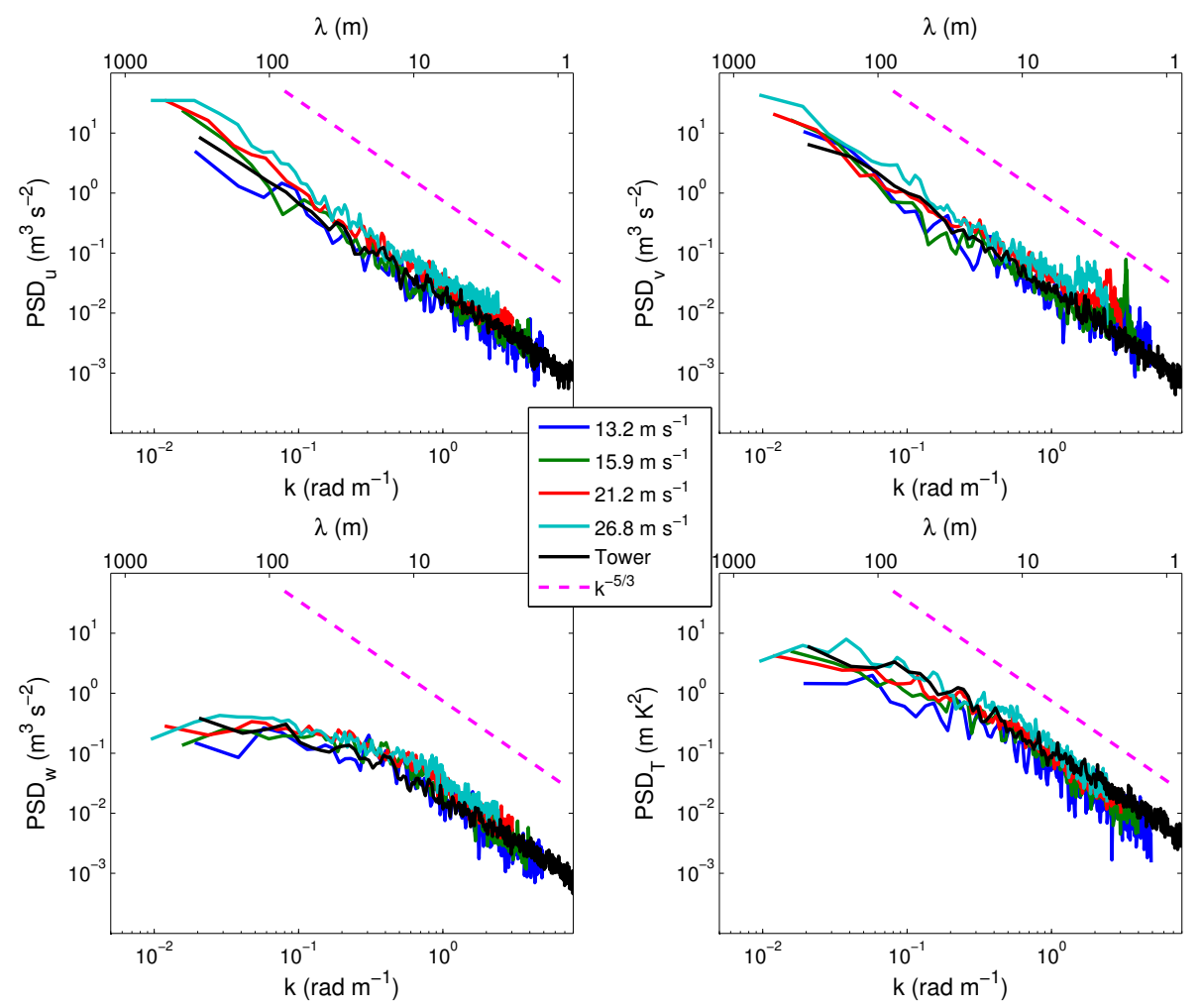

Figure 6. Sonic wind components and temperature car vs. tower wavenumber spectra. The car spectra are averaged over all tracks with the same car speed, yielding four groups of tracks (see Table 2), while the tower spectra are averaged over all tracks.

mean wind speed for the tower spectra assuming the applicability of the Taylor hypothesis. The car tracks are all $1 \mathrm{~min}$ long, unlike the $900 \mathrm{~m}$ from previous sections. This is to ensure the same number of data points for calculation of spectra, which simplifies the analysis and comparison. The final shapes of the spectra are not very sensitive to this choice. The mean wind speed for all tower spectra is taken to be $1.5 \mathrm{~m} \mathrm{~s}^{-1}$, which means that all tower spectra have the same wavenumber range. Individual tower spectra, corresponding to individual car tracks, look very similar for each variable, so they are averaged into a single spectrum. Since the car speeds are different for the four groups of tracks (see Table 2), the wavenumber ranges are also different. The car spectra are therefore averaged for each group, yielding four spectra for each variable.

Figure 6 shows the spectral agreement between the car and tower measurements. The car spectra for $u, w$ and $T$ agree well with the tower, as well as with the $k^{-5 / 3}$ law, which implies that the standard corrections work well. The spectral amplitudes slightly increase with the car speed. Since the car speed increases with successive runs, this almost certainly results from the small increase in variance due to the increasing mixing with time, as seen for example from $\overline{T^{\prime 2}}$ in Fig. 4. The spectra for the $v$ component show distinct peaks at large wavenumbers. These peaks are a function of the car speed in the wavenumber domain, which points to their local origin as frequency oscillations rather than spatial modes.

Figure 7 shows the lateral velocity spectra in the frequency domain for the car-measured wind and the GPS-INSmeasured motion. The lateral component spectra are approximately the same as the northward $v$ component for this experiment because the car track orientation has only a small deviation from the east-west direction. The characteristic peaks at about $7 \mathrm{~Hz}$ for the lateral component are at the same frequency for wind and GPS-INS, and are also at approximately the same frequency for all car speeds. This implies that these spectral peaks are caused by the frame vibrations. The reason why these vibrations are not removed after the standard corrections, even though the GPS-INS recorded them, is found in the phase shift between the wind and GPS-INS. The phase shift should be $180^{\circ}$, but is variable in the data. This is because the spectral peaks are so close to the sampling frequency that the phase shift is not adequately resolved. One solution of this issue could be to use a higher sampling rate, but the throughput of the GPS-INS does not currently allow that. Another approach is to apply a cut-off filter to remove the high frequencies where spurious oscillations occur.

Frequency spectra for all three components in the car coordinate system (Figs. 7-9) also illustrate the relative importance of the frame motion and attitude corrections. A concern was that high-wavenumber road irregularities could 

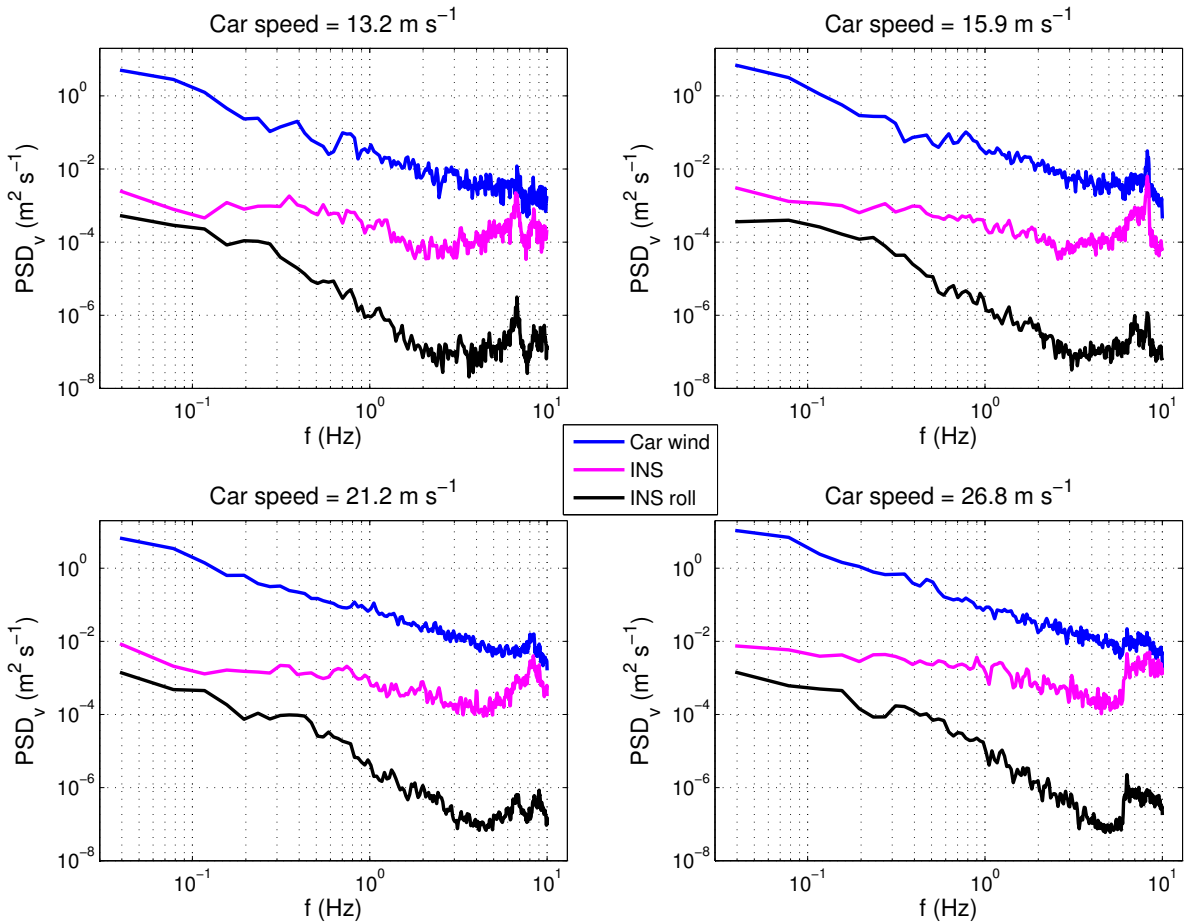

Figure 7. Frequency spectra of the lateral component (in the car coordinate system) for the car sonic and the GPS-INS motion, as well as the contribution of the roll angle to the lateral component. The latter is overestimated as the product of the roll angle and mean wind speed of $1.5 \mathrm{~m} \mathrm{~s}^{-1}$ (recall that the GPS-INS and sonic coordinate systems are aligned; Sect. 2.2). The spectra are averaged over all tracks with the same car speed (see Table 2), yielding the four panels.
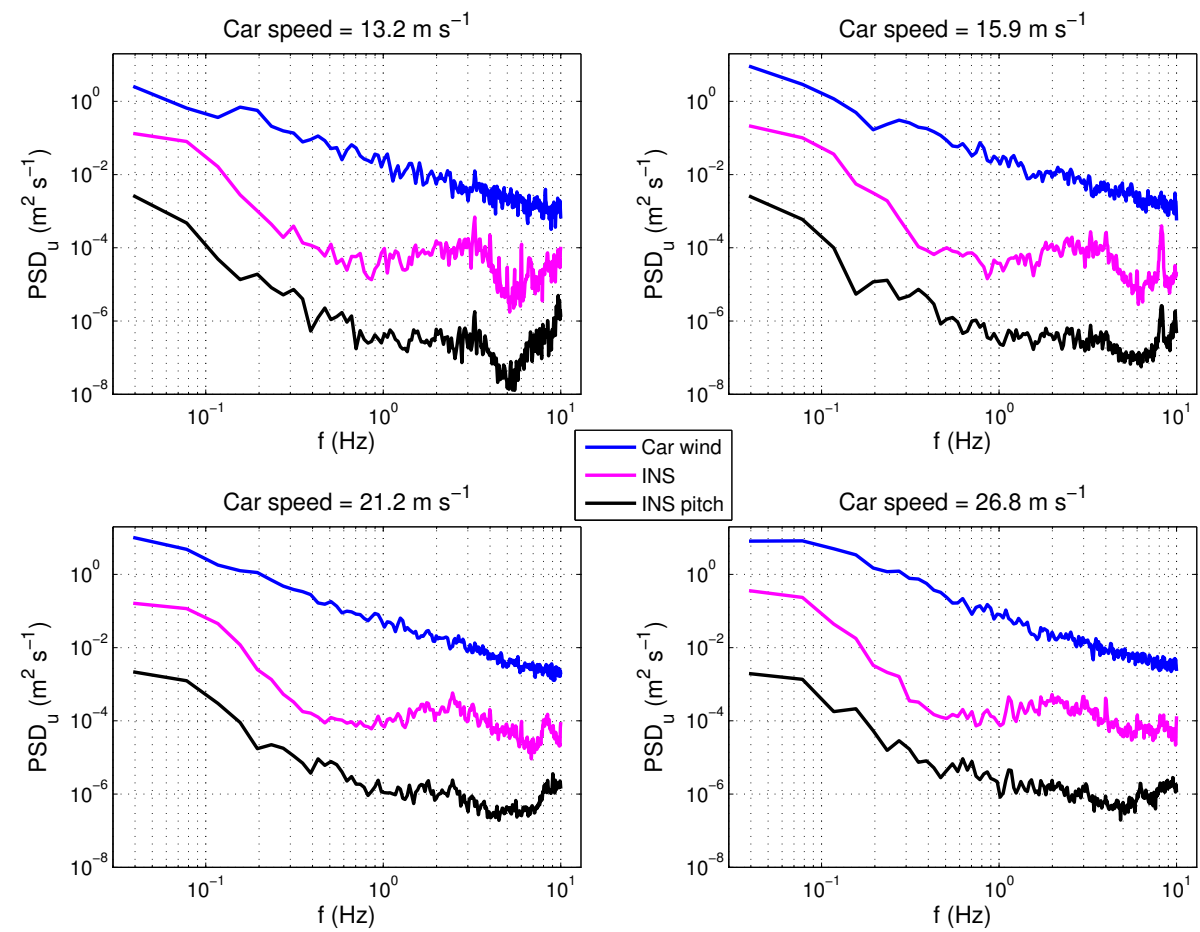

Figure 8. As in Fig. 7, except for the longitudinal component and the contribution of pitch to the longitudinal component. 

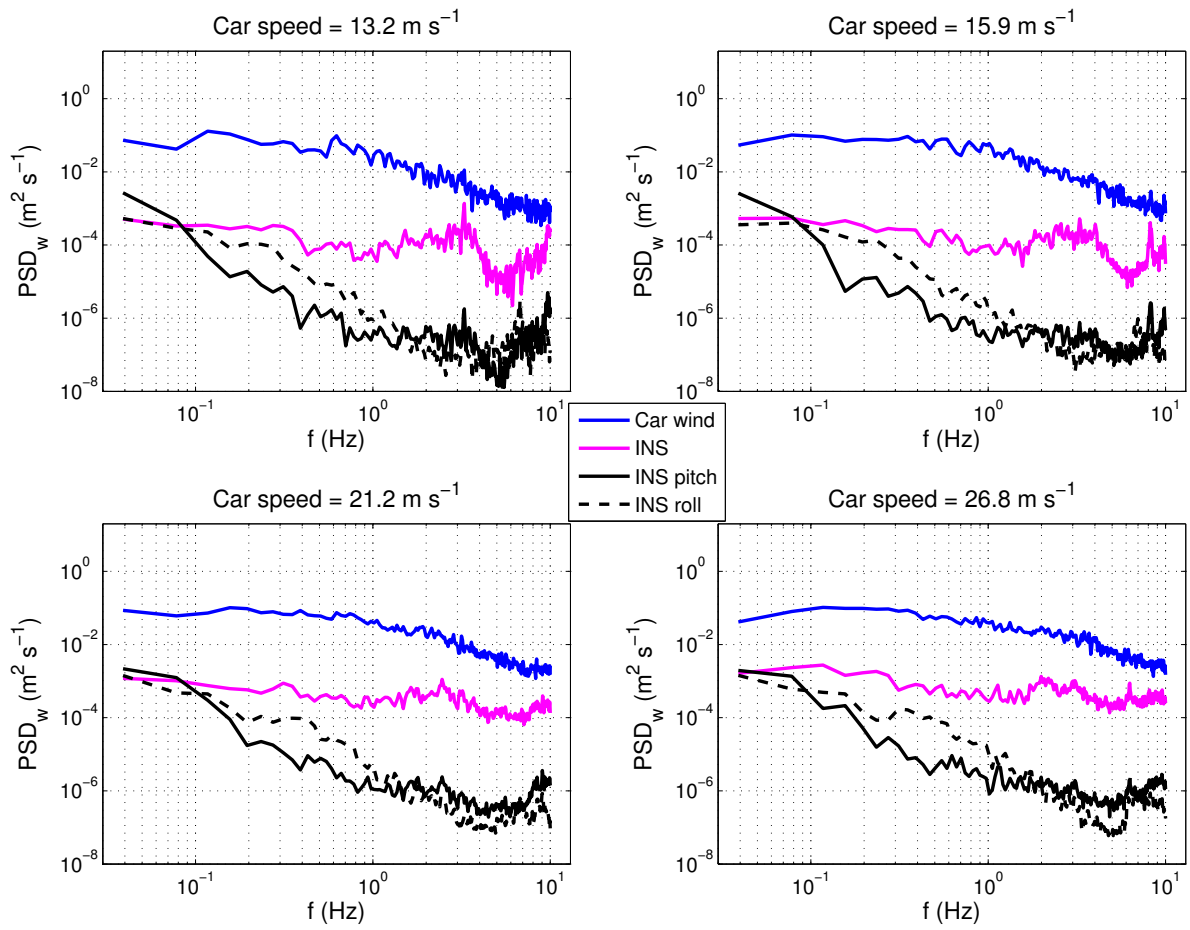

Figure 9. As in Fig. 7, except for the vertical component and the contributions of both pitch and roll to the vertical component.

introduce spurious contributions to the final wind because the three-dimensional rotation is not used to correct for the attitude angles. The spectra show that their contributions at the high-wavenumber end are at least an order of magnitude smaller than the motion of the system as recorded by the GPS-INS, which translates into being at least three times smaller for velocity. The spectra of GPS-INS motion itself are about an order of magnitude smaller than the measured wind. This implies that the most important corrections for the instrumented car are to remove the mean car speed and the mean vertical speed induced by the flow distortion. Other corrections have small to negligible effects on the final results for typical road conditions. This is substantially different from the aircraft measurements, where both the attitude angles and the aircraft motion vector are significant compared to the wind vector, and must be measured and removed to get the true wind.

\subsection{Speed bumps}

The three-dimensional rotation of the wind vector into the Earth coordinate frame (see Sect. 2.2) was used for correcting the wind when driving over the speed bumps. The tracks were divided into the parts when the car is crossing a speed bump, and the other, flat parts. The variance of $w$ when the car was crossing the speed bumps is $1.306 \mathrm{~m}^{2} \mathrm{~s}^{-2}$ after the corrections and is compared to
- the variance before the corrections, $1.554 \mathrm{~m}^{2} \mathrm{~s}^{-2}$

- the variance of the flat parts of the tracks, $1.302 \mathrm{~m}^{2} \mathrm{~s}^{-2}$.

So, the applied corrections successfully decrease the $w$ variance over the speed bumps to the value of $w$ variance over the flat road. This implies that for tracks over bumpy roads, it may be advisable to use the full three-dimensional rotation.

\section{Conclusions}

The instrumented car presented in this study is a feasible alternative and complement to towers and aircraft for measuring the ABL mean and turbulence structure. The corrections for obtaining the wind vector are smaller and simpler than for the aircraft because of the smaller amplitude and fewer degrees of freedom. Unlike for the aircraft, the corrections for pitch and roll are negligible at high frequencies, while the lateral and vertical motions of the instruments are about three times smaller than the corresponding wind components even for low-wind speeds. The crucial corrections and transformations that have to be performed are removing the longitudinal car speed, removing the mean vertical speed induced by the flow distortion, and rotating the horizontal wind to the meteorological coordinate system.

The natural simplicity and low cost of operating such a system make it a useful tool for studying near-surface turbulence statistics such as fluxes and variances. The range of applications is limited only by the presence of a road, which 
can also be relaxed if the instrumented frame is mounted on a four-wheel-drive vehicle. Studies such as horizontal mapping of the nocturnal stable ABL, probing the stable cold pool structure, and measuring horizontal gradients at the edges of forests, within forests or in urban city canyons are not possible with an aircraft. A possible alternative - a dense network of towers - is likely to be considerably more expensive and less flexible than an instrumented car.

It should be pointed out that there are more demanding meteorological situations than what we encountered here for these tests. For example, in stable weak-wind situations, the intermittent and weak nature of turbulence and smaller fluxes may significantly reduce the accuracy of the system described here for flux measurement. However, the mean and turbulence horizontal structure should still be adequately measured. For such situations involving more demanding measurement requirements, additional tests of the system performance are recommended. We also point out that there are improvements that can be made to the system, such as a more accurate and responsive INS-GPS system that can improve the system accuracy and time response, and the calculation of the footprint and disaggregation of fluxes (Hutjes et al., 2010; Metzger et al., 2013) that can quantify the relative contribution of the road to the car fluxes.

\section{The Supplement related to this article is available online at doi:10.5194/amt-7-1825-2014-supplement.}

Acknowledgements. We thank Darren Hocking for his help with the design and construction of the frame and field tests, and Larry Mahrt, Peter Isaac, Yanfei Kang and Kais Hamza for useful discussions. Helpful comments provided by Jens Bange, Thomas Foken, Alexander Moravek and Joachim Reuder are much appreciated. This study was funded by an ECR grant from Faculty of Science, Monash University. The National Center for Atmospheric Research (NCAR) is sponsored by the National Science Foundation (NSF).

Edited by: F. X. Meixner

\section{References}

Belušić, D. and Mahrt, L.: Is geometry more universal than physics in atmospheric boundary layer flow?, J. Geophys. Res., 117, D09115, doi:10.1029/2011JD016987, 2012.

Burns, S. P., Horst, T. W., Jacobsen, L., Blanken, P. D., and Monson, R. K.: Using sonic anemometer temperature to measure sensible heat flux in strong winds, Atmos. Meas. Tech., 5, 2095-2111, doi:10.5194/amt-5-2095-2012, 2012.

Foken, T., Leuning, R., Oncley, S. R., Mauder, M., and Aubinet, M.: Corrections and Data Quality Control, in: Eddy Covariance: A Practical Guide to Measurement and Data Analysis, edited by: Aubinet, M., Vesala, T., and Papale, D., 85-131, Springer, Dordrecht, Netherlands, 2012.
Gordon, M., Staebler, R. M., Liggio, J., Makar, P., Li, S.-M., Wentzell, J., Lu, G., Lee, P., and Brook, J. R.: Measurements of Enhanced Turbulent Mixing near Highways, J. Appl. Meteorol. Clim., 51, 1618-1632, doi:10.1175/JAMC-D-11-0190.1, 2012.

Hsieh, C.-I., Katul, G., and Chi, T.-W.: An approximate analytical model for footprint estimation of scalar fluxes in thermally stratified atmospheric flows, Adv. Water Resour., 23, 765-772, doi:10.1016/S0309-1708(99)00042-1, 2000.

Hutjes, R., Vellinga, O., Gioli, B., and Miglietta, F.: Disaggregation of airborne flux measurements using footprint analysis, Agr. Forest Meteorol., 150, 966-983, doi:10.1016/j.agrformet.2010.03.004, 2010.

Isaac, P., Mcaneney, J., Leuning, R., and Hacker, J.: Comparison of Aircraft and Ground-Based FluxMeasurements during OASIS95, Bound.-Lay. Meteorol., 110, 39-67, doi:10.1023/A:1026002301152, 2004.

Kaimal, J. and Gaynor, J.: Another look at sonic thermometry, Bound.-Lay. Meteorol., 56, 401-410, doi:10.1007/BF00119215, 1991.

Kang, S.-L., Lenschow, D., and Sullivan, P.: Effects of Mesoscale Surface Thermal Heterogeneity on Low-Level Horizontal Wind Speeds, Bound.-Lay. Meteorol., 143, 409-432, doi:10.1007/s10546-011-9691-4, 2012.

Lenschow, D. H. (Ed.): Probing the Atmospheric Boundary Layer, American Meteorological Society, Boston, MA, USA, 1986.

Lenschow, D. H.: Micrometeorological techniques for measuring biosphere-atmosphere trace gas exchange, in: Biogenic Trace Gases: Measuring Emissions from Soil and Water, edited by: Matson, P. A. and Harriss, R. C., 126-163, Blackwell Science, Cambridge, MA, USA, 1995.

Lenschow, D. H. and Stankov, B. B.: Length Scales in the Convective Boundary Layer, J. Atmos. Sci., 43, 1198-1209, doi:10.1175/1520-0469(1986)043<1198:LSITCB>2.0.CO;2, 1986.

Lenschow, D. H., Mann, J., and Kristensen, L.: How Long Is Long Enough When Measuring Fluxes and Other Turbulence Statistics?, J. Atmos. Ocean. Tech., 11, 661-673, doi:10.1175/15200426(1994)011<0661:HLILEW>2.0.CO;2, 1994.

Li, M., Babel, W., Tanaka, K., and Foken, T.: Note on the application of planar-fit rotation for non-omnidirectional sonic anemometers, Atmos. Meas. Tech., 6, 221-229, doi:10.5194/amt-6-221-2013, 2013.

Mahrt, L.: Flux Sampling Errors for Aircraft and Towers, J. Atmos. Ocean. Tech., 15, 416-429, doi:10.1175/15200426(1998)015<0416:FSEFAA>2.0.CO;2, 1998.

Mahrt, L., Thomas, C., Richardson, S., Seaman, N., Stauffer, D., and Zeeman, M.: Non-stationary Generation of Weak Turbulence for Very Stable and Weak-Wind Conditions, Bound.-Lay. Meteorol., 147, 179-199, doi:10.1007/s10546-012-9782-x, 2013.

Mann, J. and Lenschow, D. H.: Errors in airborne flux measurements, J. Geophys. Res., 99, 14519-14526, doi:10.1029/94JD00737, 1994.

Martin, S., Bange, J., and Beyrich, F.: Meteorological profiling of the lower troposphere using the research UAV "M2AV Carolo", Atmos. Meas. Tech., 4, 705-716, doi:10.5194/amt-4-705-2011, 2011.

Martínez, D., Jiménez, M., Cuxart, J., and Mahrt, L.: Heterogeneous Nocturnal Cooling in a Large Basin Under Very Stable Condi- 
tions, Bound.-Lay. Meteorol., 137, 97-113, doi:10.1007/s10546010-9522-z, 2010.

Mayer, S., Sandvik, A., Jonassen, M., and Reuder, J.: Atmospheric profiling with the UAS SUMO: a new perspective for the evaluation of fine-scale atmospheric models, Meteorol. Atmos. Phys., 116, 15-26, doi:10.1007/s00703-010-0063-2, 2012.

Mayr, G. J., Vergeiner, J., and Gohm, A.: An Automobile Platform for the Measurement of Foehn and Gap Flows, J. Atmos. Ocean. Tech., 19, 1545-1556, doi:10.1175/15200426(2002)019<1545:AAPFTM>2.0.CO;2, 2002.

Metzger, S., Junkermann, W., Butterbach-Bahl, K., Schmid, H. P., and Foken, T.: Measuring the 3-D wind vector with a weightshift microlight aircraft, Atmos. Meas. Tech., 4, 1421-1444, doi:10.5194/amt-4-1421-2011, 2011.

Metzger, S., Junkermann, W., Mauder, M., Butterbach-Bahl, K., Trancón y Widemann, B., Neidl, F., Schäfer, K., Wieneke, S., Zheng, X. H., Schmid, H. P., and Foken, T.: Spatially explicit regionalization of airborne flux measurements using environmental response functions, Biogeosciences, 10, 2193-2217, doi:10.5194/bg-10-2193-2013, 2013.

Patton, E. G., Sullivan, P. P., and Moeng, C.-H.: The Influence of Idealized Heterogeneity on Wet and Dry Planetary Boundary Layers Coupled to the Land Surface, J. Atmos. Sci., 62, 20782097, doi:10.1175/JAS3465.1, 2005.

Raab, T. and Mayr, G.: Hydraulic Interpretation of the Footprints of Sierra Nevada Windstorms Tracked with an Automobile Measurement System, J. Appl. Meteorol. Clim., 47, 2581-2599, doi:10.1175/2008JAMC1675.1, 2008.

Schmitt, K. F., Friehe, C. A., and Gibson, C. H.: Structure of Marine Surface Layer Turbulence, J. Atmos. Sci., 36, 602-618, doi:10.1175/1520-0469(1979)036<0602:SOMSLT>2.0.CO;2, 1979.

Schotanus, P., Nieuwstadt, F., and Bruin, H.: Temperature measurement with a sonic anemometer and its application to heat and moisture fluxes, Bound.-Lay. Meteorol., 26, 81-93, doi:10.1007/BF00164332, 1983.
Smith, S., Brown, A., Vosper, S., Murkin, P., and Veal, A.: Observations and Simulations of Cold Air Pooling in Valleys, Bound.Lay. Meteorol., 134, 85-108, doi:10.1007/s10546-009-9436-9, 2010.

Straka, J. M., Rasmussen, E. N., and Fredrickson, S. E.: A Mobile Mesonet for Finescale Meteorological Observations, J. Atmos. Ocean. Tech., 13, 921-936, doi:10.1175/15200426(1996)013<0921:AMMFFM>2.0.CO;2, 1996.

Tjernström, M. and Friehe, C. A.: Analysis of a Radome AirMotion System on a Twin-Jet Aircraft for Boundary-Layer Research, J. Atmos. Ocean. Tech., 8, 19-40, doi:10.1175/1520 0426(1991)008<0019:AOARAM>2.0.CO;2, 1991.

van den Kroonenberg, A., Martin, T., Buschmann, M., Bange, J., and Vörsmann, P.: Measuring the Wind Vector Using the Autonomous Mini Aerial Vehicle $\mathrm{M}^{2} \mathrm{AV}$, J. Atmos. Ocean. Tech., 25, 1969-1982, doi:10.1175/2008JTECHA1114.1, 2008.

Vellinga, O. S., Dobosy, R. J., Dumas, E. J., Gioli, B., Elbers, J. A., and Hutjes, R. W. A.: Calibration and Quality Assurance of Flux Observations from a Small Research Aircraft, J. Atmos. Ocean. Tech., 30, 161-181, doi:10.1175/JTECH-D-11-00138.1, 2013.

Vickers, D. and Mahrt, L.: Quality Control and Flux Sampling Problems for Tower and Aircraft Data, J. Atmos. Ocean. Tech., 14, 512-526, doi:10.1175/15200426(1997)014<0512:QCAFSP>2.0.CO;2, 1997.

Wilczak, J., Oncley, S., and Stage, S.: Sonic Anemometer Tilt Correction Algorithms, Bound.-Lay. Meteorol., 99, 127-150, doi:10.1023/A:1018966204465, 2001.

Williams, A. and Marcotte, D.: Wind Measurements on a Maneuvering Twin-Engine Turboprop Aircraft Accounting for Flow Distortion, J. Atmos. Ocean. Tech., 17, 795-810, doi:10.1175/1520-0426(2000)017<0795:WMOAMT>2.0.CO;2, 2000 . 